\title{
Twenty-Second versus Sixty-Second Dilation Duration in Endoscopic Papillary Balloon Dilation for the Treatment of Small Common Bile Duct Stones: A Prospective Randomized Controlled Multicenter Trial
}

\author{
Byoung Wook Bang', Tae Hoon Lee ${ }^{2}$, Tae Jun Song ${ }^{3}$, Joung-Ho Han ${ }^{4}$, Hyun Jong Choi ${ }^{5}$, Jong Ho Moon ${ }^{5}$, \\ Chang-II Kwon ${ }^{6}$ and Seok Jeong ${ }^{1}$ \\ ${ }^{1}$ Division of Gastroenterology, Department of Internal Medicine, Inha University School of Medicine, Incheon, ${ }^{2}$ Division of Gastroenterology, \\ Department of Internal Medicine, Soonchunhyang University Cheonan Hospital, Soonchunhyang University College of Medicine, Cheonan, \\ ${ }^{3}$ Department of Internal Medicine, Inje University Ilsan Paik Hospital, Inje University College of Medicine, Goyang, ${ }^{4}$ Department of Internal Medicine, \\ Chungbuk National University College of Medicine, Cheongju, ${ }^{5}$ Digestive Disease Center and Research Institute, Department of Internal Medicine, \\ Soonchunhyang University Bucheon Hospital, Soonchunhyang University College of Medicine, Bucheon, ${ }^{6}$ Digestive Disease Center, CHA Bundang \\ Medical Center, CHA University, Seongnam, Korea
}

See commentary on page 6-7

\begin{abstract}
Background/Aims: Endoscopic papillary balloon dilation (EPBD) has been advocated as an alternative therapy to endoscopic sphincterotomy for the treatment of common bile duct (CBD) stones. However, there is no established consensus on the optimal balloon dilation duration (BDD). We prospectively evaluated the efficacy and post-endoscopic retrograde cholangiopancreatography (ERCP) complications between the 20- and 60-second EPBD groups.

Methods: A total of 228 patients with small CBD stones $(\leq 12 \mathrm{~mm})$ were randomly assigned to undergo EPBD with a 20- or 60-second duration at six institutions. We evaluated baseline patient characteristics, endoscopic data, clinical outcomes, and procedure-related complications. In addition, we analyzed risk factors for postprocedural pancreatitis.

Results: CBD stones were removed successfully in 107 of 109 patients $(98.1 \%)$ in the 20-second group and in 112 of 119 patients (94.1\%) in the 60-second group ( $p=0.146$ ). Post-ERCP pancreatitis developed in seven patients (6.4\%) in the 20-second group and nine patients $(7.5 \%)$ in the 60 -second group $(p=0.408)$. In multivariate analysis, contrast dye injection into the pancreatic duct is a significant risk factor for post-EPBD pancreatitis.

Conclusions: Based on the data showing that there were no significant differences in safety and efficacy between the two BDD groups, 20 seconds of BDD may be adequate for treatment of small CBD stones with EPBD.
\end{abstract}

Key Words: Cholangiopancreatography; Endoscopic retrograde; Choledocholithiasis; Pancreatitis

\section{INTRODUCTION}

Endoscopic papillary balloon dilation (EPBD) was introduced in 1983 as an alternative method to endoscopic sphincterotomy (EST). ${ }^{1}$ Because this technique involves dilation of the papilla without incision, it may preserve the function of the sphincter of Oddi ${ }^{2,3}$ and can be performed safely in patients with coagulopathy and liver cirrhosis. ${ }^{4}$ In addition, $\mathrm{EPBD}$ is a technically simple procedure, and is useful for patients with altered or difficult biliary anatomy. ${ }^{5,6}$ Therefore,

Received: December 5, 2013 Revised: February 20, 2014 Accepted: March 3, 2014

Correspondence: Seok Jeong

Division of Gastroenterology, Department of Internal Medicine, Inha University Hospital, Inha University School of Medicine, 27 Inhang-ro, Jung-gu, Incheon 400-711, Korea Tel: +82-32-890-2548, Fax: +82-32-890-2549, E-mail: inos@inha.ac.kr

(c) This is an Open Access article distributed under the terms of the Creative Commons Attribution Non-Commercial License (http://creativecommons.org/licenses/by$\mathrm{nc} / 3.0$ ) which permits unrestricted non-commercial use, distribution, and reproduction in any medium, provided the original work is properly cited. 
EPBD has been widely performed, and some studies comparing efficacy and safety between EPBD and EST for the treatment of common bile duct (CBD) stones have reported similar results. ${ }^{7-9}$ However, many studies have reported a higher risk of post-endoscopic retrograde cholangiopancreatography (ERCP) pancreatitis with EPBD than with EST. ${ }^{10-13}$ Therefore, the routine use of EPBD is controversial. The mechanism of post-EPBD pancreatitis is poorly understood. However, it is likely associated with damage to the papilla during balloon dilation. Thus, the dilation method used may play a role in the development of pancreatitis. ${ }^{14}$ Some studies have explored the relationship between balloon dilation duration (BDD) and post-EPBD pancreatitis. ${ }^{14-16}$ In a retrospective study conducted in Japan, the use of shorter 15-second EPBD tended to result in reduced post-ERCP pancreatitis compared with that in 2-minute EPBD, and the severity of pancreatitis in the 15-second EPBD group was milder than that in the 2-minute EPBD group. ${ }^{14}$ Previously, we conducted a prospective comparative study of 20- and 60-second EPBD for the treatment of CBD stones. ${ }^{15}$ Our hypothesis was that 20-second EPBD may be sufficient to achieve adequate papilla dilation because the balloon waist usually disappeared within 15 seconds after achieving the maximal inflation pressure for the target diameter of the balloon. No difference in the rates of successful CBD stone extraction and post-ERCP pancreatitis were observed between the two groups, which was consistent with our hypothesis. However, our previous study had limitations of low case volume and heterogeneous balloon size (range, 6 to $18 \mathrm{~mm}$ ), and it was therefore difficult to draw any definite conclusions. A recent prospective randomized study conducted in Taiwan ${ }^{16}$ reported that longer 5-minute EPBD improved the efficacy of stone extraction and reduced the risk of pancreatitis, a result that was not consistent with those of previous studies. ${ }^{14,15}$ The authors hypothesized that longer duration of EPBD resulted in greater loosening of the sphincter of Oddi, which then led to a reduction in the risk of pancreatitis. Because of conflicting past results, we conducted a prospective multi-center randomized study in order to determine the optimal BDD for the treatment of CBD stones.

\section{MATERIALS AND METHODS}

\section{Patients}

This was a prospective, multicenter study conducted at six tertiary hospitals from September 2011 to February 2013. Patients who had been diagnosed with a CBD stone $(\leq 12$ $\mathrm{mm}$ ) using imaging studies such as abdomen computed tomography and endoscopic ultrasonography were randomly allocated to the 20- or 60-second group. Exclusion criteria were no stone on cholangiography, large CBD stones $(>12$ $\mathrm{mm}$ ), prior EST or EPBD, acute pancreatitis, choledochoduodenal fistula, benign CBD stricture, or concomitant pancreatic or biliary malignancies. Patients and procedure-related data were recorded prospectively by endoscopists on a detailed data collection sheet at the time of ERCP. All patients provided written informed consent before enrolling in the study, and the Institutional Review Board of all participating institutions approved the study.

\section{Endoscopic procedure}

The initial diagnostic ERCP provided measurements of the CBD diameter, number of stones, and maximal transverse diameter of the largest stone. Technical success of EPBD was defined as the complete removal of all CBD stones within two sessions of ERCP, regardless of the use of mechanical lithotripsy. If stone extraction with EPBD alone failed, additional EST was performed for rescue. Physical examination and measurement of serum amylase/lipase, total bilirubin, and alkaline phosphatase levels, and complete blood counts were performed at 4 and 24 hours after ERCP for earlier detection of ERCP-related complications. ${ }^{17}$

The target diameter was determined based on the bile duct and maximal stone diameter. The balloon diameter ranged from 6 to $10 \mathrm{~mm}$. BDD was defined as the period between achieving maximum inflation pressure for the target diameter and starting to deflate the balloon. The balloon was inflated gradually with diluted radiopaque contrast fluid until the target pressure was achieved, and the pressure was maintained randomly for either 20 or 60 seconds. The sphincter was judged to be adequately dilated if the balloon waist had disappeared completely on fluoroscopic examination. If the balloon waist had not disappeared within 20 seconds after achieving the maximum inflation pressure for the target diameter, we considered this a sign of biliary stricture and excluded this procedure from the analysis. After dilation of the papilla, stones were extracted using a basket catheter, a retrieval balloon catheter, or a combination of both. Mechanical lithotripsy was used when stone extraction was not successful using a conventional method. A prophylactic pancreatic stent was used when post-procedural pancreatitis was strongly expected. A balloon-occlusion cholangiogram was obtained at the end of the procedure to ensure complete stone extraction.

Total procedure time and the number of pancreatic duct injections were recorded. Procedure time was classified into cannulation time (time at start of cannulation to time at introduction of catheter deep inside the $\mathrm{CBD}$ ), stone removal time (time at completion of cannulation to the time of extraction of all CBD stones), and additional time (time at completion of extraction of CBD stones to the time of endoscope removal from the body). 
The primary outcome measurements were the frequency and severity of post-ERCP pancreatitis. Major complications associated with ERCP were classified, and their severities were graded according to the modified Cotton criteria. ${ }^{17}$ Bleeding was recorded only when there was clinical evidence of bleeding, such as melena or hematemesis, or when there was need for blood transfusion. Post-ERCP pancreatitis was defined as abdominal pain with a serum amylase level exceeding three times the normal level. The severity of pancreatitis was determined according to length of hospital stay. Asymptomatic hyperamylasemia was defined as an asymptomatic condition with serum amylase level exceeding three times the upper limit of the normal range. The secondary outcomes were complete stone extraction rate and overall

Table 1. Demographic and Baseline Characteristics

\begin{tabular}{lccc}
\multicolumn{1}{c}{ Characteristic } & $\begin{array}{c}\text { 20-Second } \\
\text { group }\end{array}$ & $\begin{array}{c}\text { 60-Second } \\
\text { group }\end{array}$ & p-value \\
& $(n=109)$ & $(n=119)$ & \\
\hline Age, yr & $62.0 \pm 16.9$ & $63.7 \pm 16.6$ & 0.457 \\
Sex, male/female & $58 / 51$ & $74 / 45$ & 0.182 \\
Jaundice & $46(42.2)$ & $53(44.5)$ & 0.789 \\
Renal failure & $2(1.8)$ & $1(0.8)$ & 0.607 \\
Liver cirrhosis & $5(4.6)$ & $5(4.2)$ & 0.597 \\
Gastrectomy & & & 0.498 \\
Bilroth I & $3(2.8)$ & $7(5.9)$ & \\
Bilroth II & $4(3.7)$ & $5(4.2)$ & \\
Malignancy & $8(7.3)$ & $16(13.4)$ & 0.194 \\
GB status & & & 0.200 \\
s/p cholecystectomy & 13 & 22 & \\
GB left in situ with stone & 77 & 71 & \\
GB left in situ without stone & 19 & 26 & \\
\hline
\end{tabular}

Values are presented as mean \pm SD or number (\%).

$\mathrm{GB}$, gallbladder; s/p, status post.
post-ERCP complications.

\section{Statistical analysis}

Referring to our previous study, ${ }^{15}$ we assumed the incidence of post-ERCP pancreatitis was $5 \%$ and $12 \%$ in the 20 and 60-second EPBD groups, respectively. The estimated sample size per group was $150(\alpha=0.05$, power $=0.80$, drop rate $=7 \%$ ).

Data analyses were performed using SPSS version 18.0 (SPSS Inc., Chicago, IL, USA). Categorical variables were compared by the chi-square test and continuous variables were analyzed using Student $t$-test. Patients were randomized according to a table of random numbers produced by a random number generator included with SPSS. Potential risk factors for post-EPBD pancreatitis were assessed by univariate logistic regression analysis, and significant predictors in univariate analysis were then included in a multivariate logistic analysis to identify risk factors for post-EPBD pancreatitis.

\section{RESULTS}

Of 243 subjects from six institutions, 15 subjects were excluded because they did not meet the inclusion criteria, and 228 subjects were finally enrolled. No significant differences in terms of baseline patient characteristics (Table 1), types of ampulla and diverticuli, and stone characteristics were observed between the two groups (Table 2). There were eight cancer patients (three stomach, two gall bladder, and three other type of cancers) in the 20-second group and 16 cancer patients (five stomach, three liver, and eight other type of cancers) in the 60-second group ( $p=0.194)$. With respect to the results of ERCP, no significant differences in overall success rate of stone extraction and time taken for stone extraction were observed between the two groups. Complete removal of

Table 2. Characteristics of Bile Duct, Stone, Ampulla, and Diverticuli

\begin{tabular}{lccc}
\hline \multicolumn{1}{c}{ Characteristic } & 20-Second group $(n=109)$ & 60 -Second group $(n=119)$ & $p$-value \\
\hline Diameter of CBD, mm & $10.6 \pm 3.0$ & $11.0 \pm 3.0$ & 0.386 \\
Mean diameter of the largest stone, mm & $6.5 \pm 2.7$ & $6.9 \pm 2.9$ & 0.119 \\
No. of stone, 1/2-3/4 or more & $60 / 26 / 23$ & $26 / 23 / 29$ & 0.543 \\
Stone classification, cholesterol/brown/black & $24 / 50 / 35$ & 61 & 0.594 \\
The shape of the ampulla & & 47 & 0.738 \\
Hemispherical & 50 & 8 \\
Papillary & 49 & 1 \\
Flat & 7 & 2 \\
Pin-point & 0 & $77 / 5 / 19 / 18$ \\
Sperical & 3 & & 0.459 \\
Type of periampullary diverticuli, none/type I/II/III & $78 / 6 / 14 / 11$ & \\
\hline
\end{tabular}

Values are presented as mean \pm SD or number.

CBD, common bile duct. 
CBD stones was achieved in 98.2\% (107/109) and 94.1\% $(112 / 119)$ in the 20 - and 60 -second BDD group, respectively $(p=0.146)$ (Table 3).

A summary of post-ERCP complications is shown in Table 4. No significant difference in the rate of post-ERCP complications was observed between the two groups. Pancreatitis occurred in seven of 109 patients $(6.4 \%)$ in the 20 -second group, and in nine of 119 patients $(7.5 \%)$ in the 60 -second group ( $p=0.408$ ). All post-ERCP pancreatitis observed in this study was mild-to-moderate, and resolved within several days.
Perforation was recognized in two patients who underwent subtotal gastrectomy with Billroth II anastomosis in the 60 -second group. In the first case, the jejunum was perforated, away from the ampulla of Vater. We thought that this might have been caused by endoscopic trauma during insertion of the endoscope into the adhesive jejunum. The lesion was successfully closed by endoscopic methods with metallic clips. In the second case, the cause of perforation was not clear. However, conservative management was successful in this patient. One patient in the 20-second group developed bleeding and pancreatitis concurrently. In the 60-second

Table 3. Results of Endoscopic Retrograde Cholangiopancreatography Procedure

\begin{tabular}{|c|c|c|c|}
\hline Group & 20 -Second group $(n=109)$ & 60 -Second group $(n=119)$ & $p$-value \\
\hline Complete CBD stone removal & $107(98.1)$ & $112(94.1)$ & 0.146 \\
\hline Total procedure time, $\min$ & $16.8 \pm 17.1$ & $17.4 \pm 8.1$ & 0.209 \\
\hline Cannulation time & $4.6 \pm 4.1$ & $4.3 \pm 3.4$ & 0.302 \\
\hline Stone removal time & $10.4 \pm 11.0$ & $11.2 \pm 5.6$ & 0.131 \\
\hline Additional time & $1.9 \pm 1.7$ & $1.8 \pm 3.1$ & 0.751 \\
\hline No. of session for complete stone removal & & & 0.052 \\
\hline One session & 106 & 107 & \\
\hline Two sessions & 3 & 12 & \\
\hline Mechanical lithotripsy & $3(2.7)$ & $3(2.5)$ & 0.476 \\
\hline Stone removal & & & 0.145 \\
\hline None & 7 & 3 & \\
\hline Basket sweeping & 14 & 7 & \\
\hline Retrieval balloon & 46 & 56 & \\
\hline Basket+retrieval & 42 & 52 & \\
\hline Injection to pancreatic duct & $23(21.1)$ & $17(14.3)$ & 0.248 \\
\hline No. of pancreatic duct cannulation, $1 / 2-3 / 4$ or more & $15 / 8 / 0$ & $11 / 6 / 0$ & 1.000 \\
\hline Degree of pancreatic duct & & & 0.354 \\
\hline Main duct & 21 & 15 & \\
\hline Branched duct & 2 & 2 & \\
\hline Use of pancreatic duct stent & 11 & 9 & 0.641 \\
\hline
\end{tabular}

Values are presented as mean \pm SD or number (\%).

CBD, common bile duct.

Table 4. Post-Endoscopic Retrograde Cholangiopancreatography Complication

\begin{tabular}{lccc}
\hline \multicolumn{1}{c}{ Variable } & 20-Second group $(n=109)$ & 60-Second group $(n=119)$ & $p$-value \\
\hline Asymptomatic hyperamylasemia & $16(14.7)$ & $12(10.1)$ & 0.685 \\
Overall post-ERCP complications & $10(9.2)$ & $13(10.9)$ & 0.826 \\
Pancreatitis & $7(6.4)$ & $9(7.5)$ & 0.408 \\
Mild, no. & 6 & 6 & 3 \\
$\quad$ Moderate, no. & 1 & $1(0.9)$ & 0.960 \\
Bleeding & $1(0.9)$ & $2(1.7)$ & 0.170 \\
Perforation & $0(0.0)$ & $2(1.7)$ & 0.619 \\
Cholangitis & $1(0.9)$ & $0(0.0)$ & 0.187 \\
Cholecystitis & $2(1.8)$ & &
\end{tabular}

Values are presented as number (\%).

ERCP, endoscopic retrograde cholangiopancreatography. 
group, one patient presented with bleeding and pancreatitis, and another patient presented with cholangitis and pancreatitis concurrently. The prevalence of asymptomatic hyperamylasemia was $14.7 \%$ in the 20 -second group and $10.1 \%$ in the 60-second group.

According to our results, BDD did not have an effect on post-ERCP pancreatitis $(p=0.432)$. We evaluated risk factors for post-ERCP pancreatitis from our data (Table 5). By univariate analysis, young age, injection into the pancreatic duct, normal diameter ( $\leq 6 \mathrm{~mm})$, and difficulty of cannulation $(\geq 5$ minutes) were significant factors $(p<0.2)$. Total procedure time, stone diameter, and BDD were not statistically significant between the two groups. However, by multivariate analysis, injection into the pancreatic duct was the only significant predictor of post-ERCP pancreatitis $(p=0.043)$.

\section{DISCUSSION}

Despite the many advantages of EPBD, post-EPBD pancreatitis has remained a controversial and serious issue., ${ }^{3,12}$ The pathogenesis of post-EPBD pancreatitis is unknown and thought to be multifactorial. Papillary edema or spasm resulting from balloon dilation, leading to obstruction of pancreatic outflow and eventual development of pancreatitis, is believed to be a mechanism of post-EPBD pancreatitis. ${ }^{18,19}$ Therefore, balloon dilation method including BDD, diameter, and pressure may differentially affect the development of post-ERCP pancreatitis.

As mentioned earlier, several studies regarding BDD and post-EPBD pancreatitis have been published. ${ }^{14-16}$ In a retrospective study conducted in Japan, ${ }^{14}$ the balloon was initially dilated for 2 minutes; however, the balloon dilation method was modified after one patient developed severe post-ERCP pancreatitis. They dilated the balloon slowly and reduced BDD to 15 seconds. They hypothesized that lower-pressured and shorter-time dilation may be less traumatic to the papilla, resulting in reduction of post-EPBD pancreatitis. By changing the balloon dilation method, pancreatitis appeared to have decreased, and severe pancreatitis was significantly reduced. However, in multivariate analysis, BDD was not statistically significant, and only pancreatic duct opacification was an independent risk factor for post-EPBD pancreatitis. ${ }^{14,19}$

Contrary to this Japanese study, one randomized trial conducted in Taiwan ${ }^{16}$ reported an association of longer duration of papillary balloon dilation with a lower rate of pancreatitis after EPBD. Their study included 170 patients with CBD stones undergoing ERCP with a $10-\mathrm{mm}$ balloon for either 1 or 5 minutes. They hypothesized that longer duration of EPBD resulted in greater loosening of the sphincter of Oddi, which led to a reduction in the rate of pancreatitis. Outcomes of patients in the 5-minute groups showed acceptable rates of procedure success (93\%) and post-EPBD pancreatitis (5\%). However, the results for the 1-minute group showed an excessively low clearance rate $(80 \%)$ with a substantially high rate of postERCP pancreatitis (15\%). It is difficult to accept the outcomes for the 1-minute EPBD group ${ }^{20}$ compared to previous EPBD studies. ${ }^{11,21}$ Nevertheless, meta-analyses of BDD reported an inverse association of duration of EPBD with pancreatitis risk, and that EPBD with an adequate duration might be better than EST because of comparable rates of pancreatitis but lower rates of overall complications. ${ }^{22}$

Two previous studies have suggested an association of BDD

Table 5. Univariate and Multivariate Analysis of Risk Factors for Post-Endoscopic Retrograde Cholangiopancreatography Pancreatitis

\begin{tabular}{|c|c|c|c|c|c|c|}
\hline \multirow{2}{*}{ Variable } & \multicolumn{3}{|c|}{ Univariate analysis } & \multicolumn{3}{|c|}{ Multivariate analysis } \\
\hline & OR & $95 \%$ CI & $p$-value & OR & $95 \% \mathrm{CI}$ & $p$-value \\
\hline Age group (under 60 years) & 2.361 & $0.893-6.245$ & 0.083 & 2.558 & $0.874-7.486$ & 0.086 \\
\hline Gender female & 1.845 & $0.699-4.868$ & 0.216 & & & \\
\hline Total bilirubin $(\leq 1.3 \mathrm{mg} / \mathrm{dL})$ & 1.234 & $0.445-3.421$ & 0.686 & & & \\
\hline Injection to pancreatic duct & 3.375 & $0.019-3.375$ & 0.019 & 3.072 & $1.035-9.112$ & 0.043 \\
\hline Ballooning time (20 vs. 60 ) & 1.484 & $0.554-3.976$ & 0.432 & & & \\
\hline Normal diameter $(\leq 6 \mathrm{~mm})$ & 3.125 & $0.612-15.966$ & 0.171 & 0.817 & $0.736-1.032$ & 0.858 \\
\hline Stone diameter & 0.953 & $0.797-1.139$ & 0.953 & & & \\
\hline Total procedure time & 1.00 & $0.999-1.001$ & 0.927 & & & \\
\hline Cannulation time & 0.999 & $0.997-1.001$ & 0.999 & & & \\
\hline Stone removal time & 1.000 & $0.999-1.002$ & 1.000 & & & \\
\hline Diverticulum & 1.076 & $0.387-2.991$ & 0.889 & & & \\
\hline Ampullary type & 1.569 & $0.729-3.376$ & 0.249 & & & \\
\hline Cannulation difficulty ( $\geq 5 \mathrm{~min}$ ) & 2.649 & $1.001-7.008$ & 0.050 & 1.224 & $0.135-11.075$ & 0.858 \\
\hline
\end{tabular}

OR, odds ratio; $\mathrm{CI}$, confidence interval. 
with post-EPBD pancreatitis, contrary to the result of the present study. In the current study, BDD did not show an association with post-EPBD pancreatitis when the waistline was adequately dilated. However, we should consider the differences in study design from previous studies, which enrolled patients with CBD stones regardless of stone size (up to 32 $\mathrm{mm}$ in the Taiwanese study, ${ }^{16}$ and up to $42 \mathrm{~mm}$ in the Japanese study $\left.{ }^{14}\right)$, and used fixed balloon diameters ( $8 \mathrm{~mm}$ in Japan, and $10 \mathrm{~mm}$ in Taiwan) regardless of stone size and CBD diameter. On the other hand, we included patients with small CBD stones $(<12 \mathrm{~mm}$ ) and selected balloon diameters (6 to $10 \mathrm{~mm}$ ) depending on the size and diameter of CBD stones. ${ }^{21}$ The main reason for failure of EPBD is insufficient dilation of CBD opening to the same extent as that in EST in patients with large stones, ${ }^{23,24}$ and papilla trauma during stone extraction through an insufficiently dilated sphincter that may increase the risk of pancreatitis. In contrast, balloon dilation larger than $\mathrm{CBD}$ diameter will increase the risk of $\mathrm{CBD}$ perforation.

In this study, the definition of BDD was the period between achieving maximal inflation pressure for the target diameter and starting to deflate the balloon. This was a little different from the definition of BDD used in more relevant articles, which is usually the period between disappearance of balloon waist and starting of balloon deflation. ${ }^{14,16,17}$ However, the balloon waist disappeared during gradual balloon inflation or several seconds after the target pressure was attained in most cases. In addition, the conventional balloon (6 to $10 \mathrm{~mm}$ ) was dilated without a balloon waist, unlike the large balloon. Therefore, the period of BDD in this study was equal to or slightly shorter than that of other studies.

In the current study, we excluded patients with large CBD stones $(>12 \mathrm{~mm}$ ) because EPBD with large balloons (12 to $20 \mathrm{~mm}$ ) has been widely used for the removal of large CBD stones. ${ }^{25-27}$ Endoscopic papillary large balloon dilation (EPLBD) may make the removal of large CBD stones easier, reducing the need for mechanical lithotripsy and shortening cannulation and stone removal times, thus decreasing the incidence of post-ERCP pancreatitis. Although data regarding EPLBD are relatively limited, overall rates of pancreatitis have been remarkably low, $2.8 \%$ (range, $0 \%$ to $8 \%)^{28-30}$ compared to those for conventional EPBD. In addition, even if some studies reported on EPLBD only, ${ }^{26,31}$ most endoscopists performed EPLBD in combination with limited EST. ${ }^{25,27}$ Therefore, it causes dilation of the papilla and tearing of the sphincter of Oddi; thus, the mechanisms of EPBD and EPLBD for the development of post-ERCP pancreatitis may be different.

In the current study, BDD did not show an association with post-EPBD pancreatitis, and injection into the pancreatic duct was the only risk factor for post-EPBD pancreatitis.
Based on previous studies, ${ }^{19,28}$ pancreatic opacification is already a well-known risk factor for ERCP-related pancreatitis. Other risk factors for post-EPBD pancreatitis have been previously investigated in several studies. Seo et al. ${ }^{32}$ compared EPBD and antegrade dilation with percutaneous transhepatic papillary balloon dilation (PTPBD), and the balloon dilation method was the same in the two groups. Pancreatitis occurred in 6.7\% (14 of 208 patients) in the EPBD group, whereas there were no cases of pancreatitis in the PTPBD group. The authors explained that post-EPBD pancreatitis may be associated with the procedures of ERCP, such as cannulation and stone extraction, rather than balloon dilation itself. Balloon size may play a role in the development of post-EPBD pancreatitis because it may influence the dilation pressure. However, Li et al. ${ }^{21}$ reported that no significant correlation was observed between post-EPBD pancreatitis and balloon size. History of pancreatitis is a known risk factor for the development of pancreatitis. ${ }^{33}$ However, we did not record this factor and therefore could not discuss this relationship. Difficulty of cannulation, which is a risk factor for post-ERCP pancreatitis, ${ }^{34}$ was not a significant predictor in either this or previous studies. ${ }^{35,36}$

This study has several limitations. First, the sample size was relatively small despite being a multicenter trial. In fact, the target sample size of this study by power calculation was 300 subjects. However, two sessions of the interim analysis during this study resulted in similar outcomes. In addition, post-ER$\mathrm{CP}$ pancreatitis was more likely to occur than reported in the published literature on EST. ${ }^{11,13}$ Therefore, we did not conduct this study until we achieved the target number. In our opinion, EPBD should be reserved for selected patients with altered anatomy or impaired hemostasis. Second, we enrolled those subjects who used pancreatic stents. In this study, which evaluated the efficacy and post-ERCP complications between two BDD groups, pancreatic stents might have acted as a major confounding factor, as they are known to decrease the risk of post-ERCP pancreatitis. ${ }^{37}$ However, the effect of using pancreatic stents would be minimal, because the number of subjects using pancreatic stents was relatively small, and the distribution of these subjects in both groups was similar.

In conclusion, based on these prospective multicenter data showing that there were no significant differences in safety and efficacy between the two BDD groups, 20 seconds of $\mathrm{BDD}$ may be adequate for the treatment of small CBD stones with EPBD. Injection into the pancreas was a risk factor for post-EPBD pancreatitis in this study.

\section{Conflicts of Interest}

The authors have no financial conflicts of interest. 


\section{Acknowledgments}

This research was supported by grants from the Korean GI Endoscopy Research Foundation.

\section{REFERENCES}

1. Staritz M, Ewe K, Meyer zum Büschenfelde KH. Endoscopic papillary dilation (EPD) for the treatment of common bile duct stones and papillary stenosis. Endoscopy 1983;15(Suppl 1):197-198.

2. Minami A, Nakatsu T, Uchida N, et al. Papillary dilation vs sphincterotomy in endoscopic removal of bile duct stones. A randomized trial with manometric function. Dig Dis Sci 1995;40:2550-2554.

3. Sato H, Kodama T, Takaaki J, et al. Endoscopic papillary balloon dilatation may preserve sphincter of Oddi function after common bile duct stone management: evaluation from the viewpoint of endoscopic manometry. Gut 1997;41:541-544.

4. Park DH, Kim MH, Lee SK, et al. Endoscopic sphincterotomy vs. endoscopic papillary balloon dilation for choledocholithiasis in patients with liver cirrhosis and coagulopathy. Gastrointest Endosc 2004;60:180185.

5. Campo Fernández de los Ríos R, Brullet Benedí E. Endoscopic treatment of choledocholithiasis in patients with Billroth II gastrectomy by dilatation with papillary balloon. Gastroenterol Hepatol 1998;21:391393.

6. Liao WC, Huang SP, Wu MS, Lin JT, Wang HP. Comparison of endoscopic papillary balloon dilatation and sphincterotomy for lithotripsy in difficult sphincterotomy. J Clin Gastroenterol 2008;42:295-299.

7. Lin CK, Lai KH, Chan HH, et al. Endoscopic balloon dilatation is a safe method in the management of common bile duct stones. Dig Liver Dis 2004;36:68-72

8. Tanaka S, Sawayama T, Yoshioka T. Endoscopic papillary balloon dilation and endoscopic sphincterotomy for bile duct stones: long-term outcomes in a prospective randomized controlled trial. Gastrointest Endosc 2004;59:614-618.

9. Natsui M, Narisawa R, Motoyama H, et al. What is an appropriate indication for endoscopic papillary balloon dilation? Eur J Gastroenterol Hepatol 2002;14:635-640.

10. Bergman JJ, Rauws EA, Fockens P, et al. Randomised trial of endoscopic balloon dilation versus endoscopic sphincterotomy for removal of bileduct stones. Lancet 1997;349:1124-1129.

11. Baron TH, Harewood GC. Endoscopic balloon dilation of the biliary sphincter compared to endoscopic biliary sphincterotomy for removal of common bile duct stones during ERCP: a metaanalysis of randomized, controlled trials. Am J Gastroenterol 2004;99:1455-1460.

12. Disario JA, Freeman ML, Bjorkman DJ, et al. Endoscopic balloon dilation compared with sphincterotomy for extraction of bile duct stones. Gastroenterology 2004;127:1291-1299.

13. Liu Y, Su P, Lin S, et al. Endoscopic papillary balloon dilatation versus endoscopic sphincterotomy in the treatment for choledocholithiasis: a meta-analysis. J Gastroenterol Hepatol 2012;27:464-471.

14. Tsujino T, Kawabe T, Isayama H, et al. Efficacy and safety of low-pressured and short-time dilation in endoscopic papillary balloon dilation for bile duct stone removal. J Gastroenterol Hepatol 2008;23:867-871.

15. Bang BW, Jeong S, Lee DH, et al. The ballooning time in endoscopic papillary balloon dilation for the treatment of bile duct stones. Korean J Intern Med 2010;25:239-245.

16. Liao WC, Lee CT, Chang CY, et al. Randomized trial of 1-minute versus 5-minute endoscopic balloon dilation for extraction of bile duct stones. Gastrointest Endosc 2010;72:1154-1162.

17. Cotton PB, Lehman G, Vennes J, et al. Endoscopic sphincterotomy complications and their management: an attempt at consensus. Gastrointest Endosc 1991;37:383-393.

18. Lee TH, Moon JH, Choi HJ, et al. Prophylactic temporary 3F pancreatic duct stent to prevent post-ERCP pancreatitis in patients with a difficult biliary cannulation: a multicenter, prospective, randomized study. Gastrointest Endosc 2012;76:578-585.

19. Tsujino T, Isayama $H$, Komatsu $Y$, et al. Risk factors for pancreatitis in patients with common bile duct stones managed by endoscopic papillary balloon dilation. Am J Gastroenterol 2005;100:38-42.

20. Attam R, Freeman ML. Endoscopic papillary balloon dilation for stone extraction: if, when, and for how long? Gastrointest Endosc 2010;72: 1163-1166.

21. Li NP, Liu JQ, Zhou ZQ, Ji TY, Cai XY, Zhu QY. Ampulla dilation with different sized balloons to remove common bile duct stones. World J Gastroenterol 2013;19:903-908.

22. Liao WC, Tu YK, Wu MS, et al. Balloon dilation with adequate duration is safer than sphincterotomy for extracting bile duct stones: a systematic review and meta-analyses. Clin Gastroenterol Hepatol 2012;10: 1101-1109.

23. Toda N, Saito K, Wada R, et al. Endoscopic sphincterotomy and papillary balloon dilation for bile duct stones. Hepatogastroenterology 2005; 52:700-704.

24. Tsujino T, Kawabe T, Komatsu Y, et al. Endoscopic papillary balloon dilation for bile duct stone: immediate and long-term outcomes in 1000 patients. Clin Gastroenterol Hepatol 2007;5:130-137.

25. Ersoz G, Tekesin O, Ozutemiz AO, Gunsar F. Biliary sphincterotomy plus dilation with a large balloon for bile duct stones that are difficult to extract. Gastrointest Endosc 2003;57:156-159.

26. Jeong S, Ki SH, Lee DH, et al. Endoscopic large-balloon sphincteroplasty without preceding sphincterotomy for the removal of large bile duct stones: a preliminary study. Gastrointest Endosc 2009;70:915-922.

27. Youn YH, Lim HC, Jahng JH, et al. The increase in balloon size to over $15 \mathrm{~mm}$ does not affect the development of pancreatitis after endoscopic papillary large balloon dilatation for bile duct stone removal. Dig Dis Sci 2011;56:1572-1577.

28. Meine GC, Baron TH. Endoscopic papillary large-balloon dilation combined with endoscopic biliary sphincterotomy for the removal of bile duct stones (with video). Gastrointest Endosc 2011;74:1119-1126.

29. Maydeo A, Bhandari S. Balloon sphincteroplasty for removing difficult bile duct stones. Endoscopy 2007;39:958-961.

30. Misra SP, Dwivedi M. Large-diameter balloon dilation after endoscopic sphincterotomy for removal of difficult bile duct stones. Endoscopy 2008;40:209-213.

31. Chan HH, Lai KH, Lin CK, et al. Endoscopic papillary large balloon dilation alone without sphincterotomy for the treatment of large common bile duct stones. BMC Gastroenterol 2011;11:69.

32. Seo YR, Moon JH, Choi HJ, et al. Papillary balloon dilation is not itself a cause of post-endoscopic retrograde cholangiopancreatography pancreatitis; results of anterograde and retrograde papillary balloon dilation. J Gastroenterol Hepatol 2013;28:1416-1421.

33. Sugiyama M, Izumisato Y, Abe N, Masaki T, Mori T, Atomi Y. Predictive factors for acute pancreatitis and hyperamylasemia after endoscopic papillary balloon dilation. Gastrointest Endosc 2003;57:531-535.

34. Freeman ML, Nelson DB, Sherman S, et al. Complications of endoscopic biliary sphincterotomy. N Engl J Med 1996;335:909-918.

35. Bergman JJ, van Berkel AM, Bruno MJ, et al. Is endoscopic balloon dilation for removal of bile duct stones associated with an increased risk for pancreatitis or a higher rate of hyperamylasemia? Endoscopy 2001; 33:416-420.

36. Sugiyama M, Abe N, Izumisato Y, Masaki T, Mori T, Atomi Y. Risk factors for acute pancreatitis after endoscopic papillary balloon dilation. Hepatogastroenterology 2003;50:1796-1798.

37. Choudhary A, Bechtold ML, Arif M, et al. Pancreatic stents for prophylaxis against post-ERCP pancreatitis: a meta-analysis and systematic review. Gastrointest Endosc 2011;73:275-282. 